\section{PSQ-094 USE OF EXCIPIENTS IN ORAL LIQUID COMMERCIAL MEDICINES IN A CHILDREN'S HOSPITAL}

D Gavrus Ion, F Bossacoma Busquets, MA Romo Murillo, JM Catala Foguet, A Diaz Cambron, M Sánchez Celma*, M Villaronga Flaqué, JA Cuartero Lozano, J Vinent Genestar, R Farre Riba. Hospital Sant Joan de Déu, Pharmacy, Esplugues de Llobregat, Spain

10.1136/ejhpharm-2019-eahpconf.527

Background Oral liquid medicines, such as solutions and suspensions, are commonly given to young children, because they are easy to swallow and allow weight-based dosage. The development of oral medicines for paediatric patients often requires age-appropriate formulations which can be more complex and may involve a broader range of excipients than adult dosage forms.

Purpose Identify excipients having a potential risk of safety concerns in the paediatric population of commercial oral liquid medicines of our hospital formulary.

Material and methods All oral liquid medicines included in the hospital's drug formulary were reviewed, using components information from the summary product characteristics obtained from the Spanish Medicament Agency (AEMPS ${ }^{1}$ ), and compared with the oral excipients from the European Commission guideline on 'Excipients in the labelling and package leaflet of medicinal products for human use'. ${ }^{2}$

Results We reviewed 96 SPC oral liquid medicines, 16 oral drops and 80 suspensions or solutions, only 12 of which were non-indicated excipients-free.

Bold excipients contraindicated in neonates/children less than 6 years. Italic: contraindicated in patients with metabolic disorders. ${ }^{3}$

\section{Abstract 5PSQ-094 Table 1}

\begin{tabular}{ll}
\hline Excipient & Percentage (\%) \\
\hline Aspartame & 4 \\
Azo colouring agents & 4 \\
Benzoic acid (E210) and benzoates & 25 \\
Benzyl alcohol & 3 \\
Cyclodextrins & 1 \\
Ethanol & 19 \\
Fructose & 5 \\
Glucose & 6 \\
Glycerol (E422) & 27 \\
Gluten & 2 \\
Sulphites including metabisulphites & 1 \\
Sucrose & 31 \\
Soya oil/hydrogenated soya oil & 3 \\
Sorbitol (E420) & 21 \\
Propylene glycol (E1520) and esters of propylene glycol & 25 \\
Phenylalanine & 1 \\
Parahydroxybenzoates and their esters & 33 \\
Mannitol (E421) & 5 \\
Maltitol (E965) & 3 \\
Macrogolglycerol ricinoleate & 13 \\
None & \\
\hline & \\
\hline
\end{tabular}

Conclusion A high percentage (87\%) of liquid medicines in our formulary commonly used to treat children contain potentially harmful excipients. So, specific criteria have to be implemented in the drug procurement process. The use of excipients in paediatric medicines is driven by functional requirements and should be justified through a risk-based assessment, considering, among others, the paediatric age group, frequency of dosing and duration of treatment and excipient concentration. Clinicians should be aware of this in prescribing appropriate treatment in this population. When a commercial medicine contains an excipient that can cause problems in paediatrics, compounding may be an appropriated solution.

\section{REFERENCES AND/OR ACKNOWLEDGEMENTS}

1. AEMPS https://www.aemps.gob.es/cima/publico/home.htm

2. https://www.ema.europa.eu/documents/scientific-guideline/annex-european-commission-guideline-excipients-labelling-package-leaflet-medicinal-productshuman en.pdf

3. Breikreutz J, Boos J. Expert Opin Drug Deliv 2007;4:37-45. No conflict of interest.

\section{PSO-095 ABSTRACT WITHDRAWN}


${ }^{1} \mathrm{P}$ Abdulrouf*, ${ }^{2} \mathrm{~B}$ Thomas, ${ }^{1} \mathrm{~W}$ Elkassem, ${ }^{3} \mathrm{M}$ Alhail. ${ }^{1}$ Hamad Medical Corporation, Assistant Director - Pharmacy Executive Office, Doha, Qatar; ${ }^{2} \mathrm{Hamad}$ Medical Corporation, Clinical Pharmacy Specialist - Pharmacy Executive Office, Doha, Qatar; ${ }^{3}$ Hamad Medical Corporation, Executive Director - Pharmacy Executive Office, Doha, Qatar

10.1136/ejhpharm-2019-eahpconf.529

Background Medication errors are major global issues adversely impacting patient safety and health outcomes. Medication safety practices are evolving rapidly. It is imperative to explore the views of the healthcare workforce, key stakeholders and their knowledge, attitude and practice towards strategies, and standards, to prevent medication errors.

Purpose To explore the key stakeholders' (e.g. policy-makers, professional leaders and managers, lead educators and trainers) views on strategies, standards, standardisation, priorities and the political landscape to promote patient safety and medication error reporting.

To explore their perceptions of processes of implementing change to routine practice to promote patient safety.

Material and methods The quantitative phase was done using a Hospital Survey on Patient Safety Culture questionnaire. Eighteen, in-depth interviews with a purposive sample of key stakeholders (e.g. policy-makers, professional leaders and managers, lead educators and trainers) were conducted using a topic guide derived from the previous phases of the study (focus group and questionnaire). Qualitative data analysis was undertaken using the Framework Approach.

Results One-thousand, six-hundred and four questionnaires were received, there were statistically significant scores in terms of age, experience (were more confident in reporting errors) $\mathrm{p}<0.001$ and profession (pharmacists were more confident) $\mathrm{p}<0.05$. The interviewed key stakeholders shared a common view that increased error reporting could significantly improve patient safety and they were also aware concerning the seriousness of under-reporting and thus building a non-punitive, fair-blame culture was imperative. Management support for patient safety was clearly evidenced during the interviews. Feedback and communication about errors was repeatedly recognised as key to promoting a culture of patient safety. The key stakeholders also recognised that the current medication error-reporting processes and systems were grossly sub-optimal in preventing or minimising medication errors.

Conclusion This study of key stakeholder perspectives has highlighted the key stakehoders' concern about the positive and negative aspects of organisational culture, and has informed the importance of the development of interventions

to promote patient safety and the sustainmability of a patient safety culture.

\section{REFERENCES AND/OR ACKNOWLEDGEMENTS}

https://journals.plos.org/plosone/article/authors?id=10.1371/ journal.pone.0204801

https://journals.plos.org/plosone/article/authors? $\mathrm{id}=10.1371$ / journal.pone.0204987

No conflict of interest.

\section{PSQ-097 TRIPLE WHAMMY INTERACTION: IMPROVING PATIENTS' SAFETY}

${ }^{1} \mathrm{~N}$ Alzueta, ${ }^{1} \mathrm{MC}$ Celaya, ${ }^{1} \mathrm{MT}$ Acin*, ${ }^{1} \mathrm{~A}$ Echeverría, ${ }^{1} \mathrm{C}$ Fontela, ${ }^{1} \mathrm{~L}$ Sanz, ${ }^{2} \mathrm{~N}$ Gómez, ${ }^{2}$ ML Fernandez, ${ }^{1} \mathrm{~A}$ López, ${ }^{1} \mathrm{~J}$ Garjón. ${ }^{1}$ Navarre Health Service, Pharmacy Subdirection, Pamplona, Spain; ${ }^{2}$ Navarre Health Service, Complejo Hospitalario de Navarra. Nephrology Department, Pamplona, Spain

\subsection{6/ejhpharm-2019-eahpconf.530}

Background Concomitant treatment with renin-angiotensin system inhibitors (ACEI/ARB), diuretics and non-steroidal antiinflammatory drugs (NSAID) has been named as triple whammy (TW). This interaction can produce acute kidney injury (AKI).

Purpose To implement a strategy in order to avoid the development of AKI due to TW interaction.

Material and methods A so-called 'Avoiding TW strategy' was implemented including the following activities: a multidisciplinary group (nephrologists, general practitioners (GP) and clinical pharmacists (CP)) was established to design the strategy; evidence on TW interaction and AKI was assessed; criteria for selection of candidates for intervention was agreed (concomitant use of ACEI/ARB, diuretics and NSAID); CP presented the programme to GPs; patients who were candidates for intervention were retrieved through an in-house developed software (OBSERVA) integrated in electronic clinical records in our region; a deprescription proposal was included in all retrieved clinical records with information about the risk of developing AKI due to the combination, suggesting the doctor to withdraw the NSAID and, if this was not possible, monitoring renal function and serum potassium levels was recommended; and valuation of NSAID withdrawal was planned.

Results The TW optimisation strategy was created and 1699 proposals were sent in August 2018. NSAID deprescription proposals were distributed among the different groups: M01AE (propionic acid derivatives): 54.3\%; M01AH (coxibs): 27.8\%; M01AB (acetic acid derivatives): 15.0\%; M01AC (oxicams): 2.7\%; M01AG (fenamates): $0.1 \%$; and M01AX (other NSAID): $0.1 \%$.

Preliminary results, 2 months after the implementation, showed that $15 \%$ of proposals were evaluated by GPs, with an acceptance rate of $82 \%$.

Conclusion Pharmacological interactions must be considered even more when they cause important morbidity such as AKI.

$\mathrm{CP}$ intervention through electronic clinical records optimises pharmacotherapy and may reduce adverse events and improve patients' safety.

\section{REFERENCES AND/OR ACKNOWLEDGEMENTS}

1. García MDP, Sánchez JG, Laso E, et al. Analysis of a design to detect triple whammy in patients with digoxin therapy. https://ejhp.bmj.com/content/23/ Suppl_1/A193.1

No conflict of interest. 